\title{
Diffusion Tensor Imaging: Structural Connectivity Insights, Limitations and Future Directions
}

\author{
Linda J. Lanyon \\ University of British Columbia \\ Canada
}

\begin{abstract}
"The brain is a monstrous, beautiful mess. Its billions of nerve cells-called neurons-lie in a tangled web that displays cognitive powers far exceeding any of the silicon machines we have built to mimic it"
\end{abstract}

William F. Allman

\section{Introduction}

Almost 40 years since the invention of magnetic resonance imaging (MRI)(Lauterbur, 1973) and just over 25 years since the first use of diffusion imaging (Le Bihan et al., 1986; Merboldt, Hanicke, \& Frahm, 1985; Taylor \& Bushell, 1985), the pace of advance of these fields and rate of publication of findings are still increasing. MRI neuroimaging delivers enticing insights into the function of the human brain. Elucidation of the networks in the brain that allow information to be processed in a hugely parallel and adaptive manner is crucial to the understanding of brain function. Functional MRI (Kwong et al., 1992; Ogawa et al., 1992) has shown that the brain is a modularised highly distributed system with components specialising in one or more types of information processing (Kanwisher, 2010), such as the network of cortical regions involved in the visual processing of faces (C. J. Fox, Iaria, \& Barton, 2008; Haxby, Hoffman, \& Gobbini, 2000; Ishai, Schmidt, \& Boesiger, 2005; Kanwisher, McDermott, \& Chun, 1997; Rossion et al., 2003). Investigation of the large-scale interconnectivity of these modules will lead to better understanding of how these functional building blocks cooperate to confer human cognition, behaviour, memory and conscious thought. Diffusion tensor imaging (DTI) is a non-invasive MRI method used to visualize white matter pathways by identifying the location and orientation of large white matter bundles, at the millimetre-level of 3D visualization. The remarkable advantage of this technique is that it provides the first opportunity to non-invasively investigate this structural connectivity in vivo in human beings.

The aim of this chapter is to provide an accessible outline of DTI methods and their use in elucidating large-scale brain connectivity. We will review the field discussing results from human DTI in health and disease, using examples to demonstrate the use of DTI in providing an indication of the structural integrity of cortical white matter, and correlation of individual structure and behavioural performance. We will describe the principles of DTI 
tractography and show how this is used to investigate and visualize white matter pathways in healthy and pathological brains. Finally, we will discuss future developments in the field, in particular directions of research that aim to overcome some of the current limitations of the technique. We begin with the principles of diffusion-weighted MRI.

\section{Foundations of the field: DW-MRI and the diffusion tensor}

DTI employs diffusion-weighted MRI (DW-MRI) scanning. MRI techniques generally observe properties of hydrogen nuclei, which consist of a single proton, and are the most dominant atom in the human body due to the abundance of water molecules. DW-MRI observes the displacement of these protons due to random Brownian motion (diffusion). Specifically, the technique measures the probability density function of proton displacements over a fixed time interval. In the presence of a strong magnetic field inside the MRI scanner, water protons are excited and begin to precess (spin), in similar fashion to a series of gyroscopes, in phase with each other. For functional MRI, a T2-weighted scan measures the time taken in different media for this precession to relax, following excitation. When molecules are able to move freely, this relaxation tends to take longer, so areas of cerebral spinal fluid (CSF) tend to appear brighter than white or grey matter and white matter tends to appear darker than grey matter. Similarly, in DW-MRI, differences in the homogeneity of the precession of water molecules are used to confer information about media type. In these scans a dephasing gradient pulse field is used to dephase the precession of molecules. The extent of dephasing varies along the gradient, as shown in figure 1. A subsequent rephasing gradient of opposite polarity restores phase to all molecules that have not moved. Molecules that have moved in the time between the two gradient pulses will not have their phase fully refocused by the rephasing pulse. By applying this pair of gradients between MRI excitation and data collection, the image is sensitised to the motion of water molecules by diffusion (or flow). The strength of diffusionweighting is often expressed as a $b$ value, which is proportional to the product of the square of the gradient strength and the time interval between the two pulses. The extent of signal decay depends on the diffusion constants of the medium. Signal from CSF decays faster and, therefore, produces a darker image than that for the rest of the brain. The signal from white matter decays the slowest and, therefore, white matter appears as the darkest regions of the image. Simple scalar apparent diffusion constant (ADC) images reflect this overall signal-loss and the acute stage of stroke can be detected from these images because swelling restricts diffusion and so the affected region appears bright (Moseley et al., 1990).

In anisotropic media such as brain white matter, a single scalar measurement of diffusion (ADC) is insufficient because the measured diffusivity depends on the orientation of the tissue. A symmetric diffusion tensor (Basser, Mattiello, \& LeBihan, 1994; Crank, 1975) is used to characterise diffusion in 3 dimensions. The tensor is usually visualised as an elliptical Gaussian. MRI scanners can measure diffusion along any desired angular direction by using in combination three independent gradient units that are orthogonal to each other. In order to create a diffusion tensor, information must be acquired from gradients applied at a minimum of 6 independent angular directions (Basser, et al., 1994), typically more are used, in addition to an image acquired without diffusion weighting (known as a b0 image). In regions where diffusion of water molecules is unhindered, such as in CSF, molecules are able to diffuse equally in all directions producing an isotropic distribution. In this case, the diffusion tensor is isotropic, depicted as a sphere. In contrast, in white matter, fiber membranes restrict diffusion in the direction perpendicular to the fibers. In fact, water 

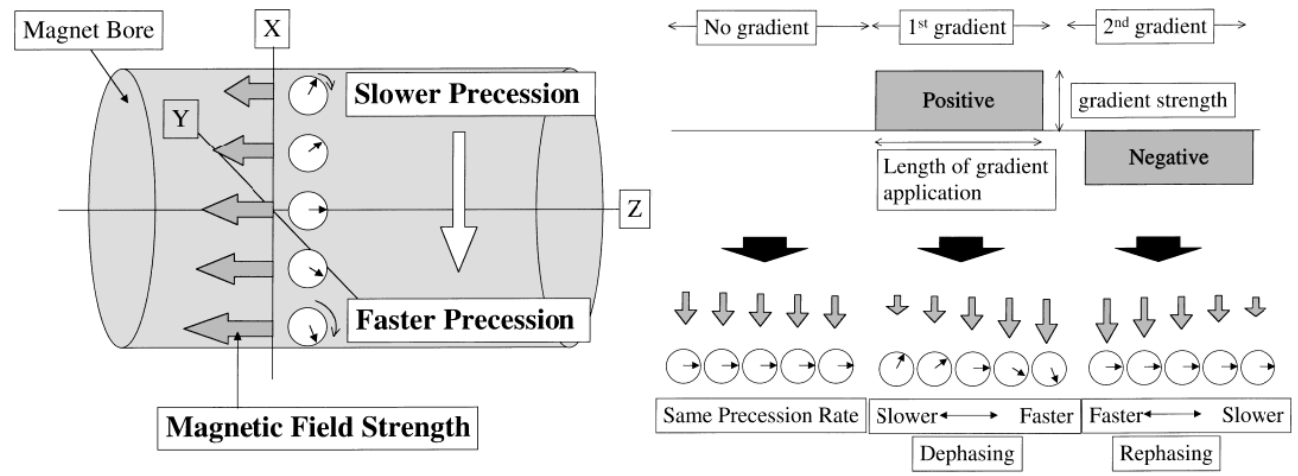

Fig. 1. Illustration of DW-MRI, from Mori and Barker (1999) figures 5 \& 6. First, a dephasing gradient pulse is applied (as shown on the left). This causes protons to lose their uniform phase (dephase) because each proton starts to precess at different rates depending on its position in space. The gradient shown on the left is applied (shown here in the $x$ direction), introducing a linear magnetic field inhomogeneity with a specified time period, magnitude, and direction. The length of grey arrows indicates the strength of the magnetic field that is non-uniform during the application of the gradients. The direction along the magnet bore (the strong static magnetic field to which the main magnetic field aligns) is defined as z. Following application of the gradient field, precession rates vary according to position of the protons within the field, as shown. After a time delay, a rephrasing gradient pulse of opposite magnitude polarity is applied (see diagram on right) and the system restores uniform phase (rephase). This rephasing is complete only when protons did not move by Brownian motion (i.e. diffuse) during the time between the application of the first and second gradients. The less complete the rephasing, the more signal loss results. (Figures reproduced with permission from John Wiley and Sons)

diffuses 3-7 times more rapidly along the length of axons aligned in white matter tracts than in the direction perpendicular to the axons (Le Bihan, 2003; Pierpaoli, Jezzard, Basser, Barnett, \& Di Chiro, 1996). This results in a tensor that is elongated, anisotropic in shape, such that the longest principle axis of the tensor reflects the main direction of diffusion in the voxel. While unmyelinated axonal membranes are sufficient to produce anisotropic diffusion (Beaulieu \& Allen, 1994), the presence of myelin further modulates anisotropy (Beaulieu, 2002) and this leads the technique to be suitable to detect a range of diseases that affect myelin. Anisotropy is higher in white than grey matter, and in adult brains compared to those of newborns. In fact, DTI has been used to quantify the development of major white matter pathways in premature infants in whom indices of anisotropy increase with age (Berman et al., 2005). The presence and direction of fiber tracts can be inferred from the diffusion tensor but, given the size of imaged voxels (typically 1-4mm for DTI) compared to the size of an axon $\left(<10 \mu \mathrm{m}\right.$, white matter packing density $\left.10^{11} \mathrm{~m}^{-2}\right)$, many collinear fibers must be present to influence overall diffusion anisotropy across the voxel. This is why DTI tractography, discussed later, typically describes the path of major white matter fiber bundles in the brain. The reader is referred to Basser and Jones (2002), Hagmann et al. (2006) and Mori and Barker (1999) for further explanation of DW-MRI. 


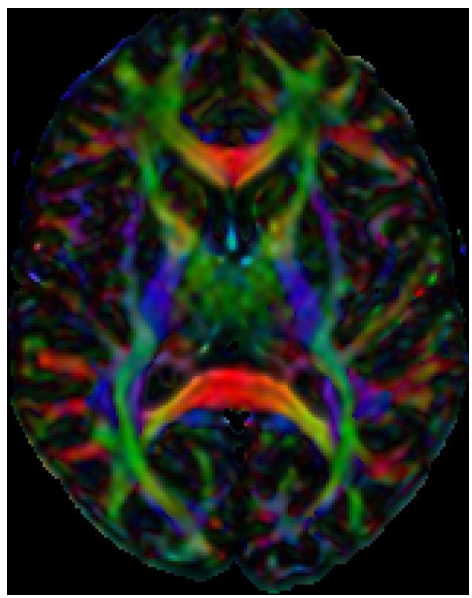

Fig. 2. Directionality-coloured anisotropy map, axial slice view. Voxels with anisotropic diffusion in the superior-inferior direction are shown in blue, anterior-posterior in green and left-right in red (for example, part of the corpus callosum)

Various scalar indices can be derived from the diffusion tensor to describe the profile of diffusion within an imaged voxel. Mean diffusivity (MD), a scalar invariant to the direction of anisotropy, describes the overall mean-squared displacement of molecules. MD has been shown to increase in certain disease states, for example in Alzheimer's disease (Agosta et al., 2011), likely reflecting a loss of white matter integrity. Of the diffusion anisotropy indices, the most commonly used is fractional anisotropy (FA), which ranges from 0 for isotropic diffusion to 1 for anisotropic diffusion. FA values across the brain are typically plotted in a colour-coded manner to form a directionality-coloured FA map, such as the one shown in figure 2. It can be seen that tensor information alone somewhat delineates white matter structure in the brain because it shows the locations and orientation of areas of high anisotropy. However, this information is based on local anisotropy in each imaged voxel. Tractography techniques, described later, consolidate this information to form a description of the pathways.

\section{Correlating structure $\&$ behaviour}

\subsection{Individual variation in the healthy population}

DTI can be used as an index of individual structural variation within the human population. Differences in DTI indices have been shown to correlate with measures of behavioural performance in both health and disease. For example, correlation between FA and behavioural performance has been found in cognitive domains such as reading ability (Klingberg et al., 2000), visuo-spatial attention (Tuch et al., 2005) and mental rotation (Wolbers, Schoell, \& Buchel, 2006). We compared FA value in the human hippocampal region with healthy individual behavioural performance during a spatial navigation task in a virtual environment (Iaria, Lanyon, Fox, Giaschi, \& Barton, 2008). The ability to spatially orient within an environment varies greatly between individuals (Ohnishi, Matsuda, Hirakata, \& Ugawa, 2006). In our experiment subjects first had to navigate within a computerised virtual environment to form a "cognitive map" (Tolman, 1948), a spatial 
memory of the environment. Next, they were asked to navigate to a specified landmark as quickly as possible using the shortest route. The task, therefore, involved both learning and retrieval components. Subjects underwent MRI scanning to obtain a high-resolution structural scan and diffusion-weighted scans using 32 gradient directions. Using these images we draw a region of interest (ROI) in the region of each subject's hippocampus, as shown in figure 3, and obtained the mean FA value within this ROI (using DTI Studio software (Hiang \& Mori)). The results revealed a statistically significant correlation between the FA in the right hippocampus and the time spent by the participants performing both the learning and retrieval tasks. Subjects with higher FA values in the area of the right hippocampus required less time to form a cognitive map (figure 4a) and were more efficient in using the map during navigation in the subsequent retrieval task (figure $4 \mathrm{~b}$ ). No similar correlations were found in the left hemisphere.

(a)

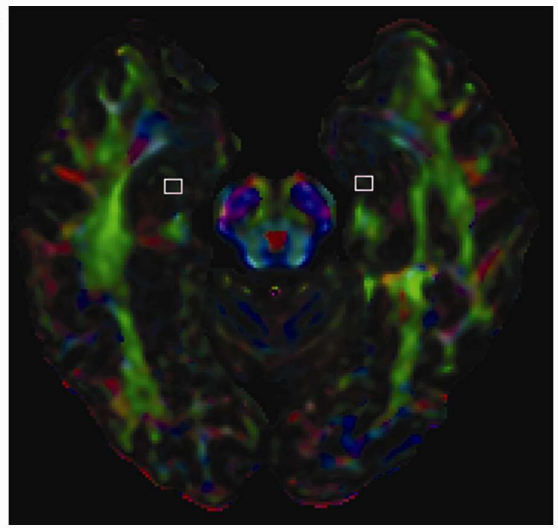

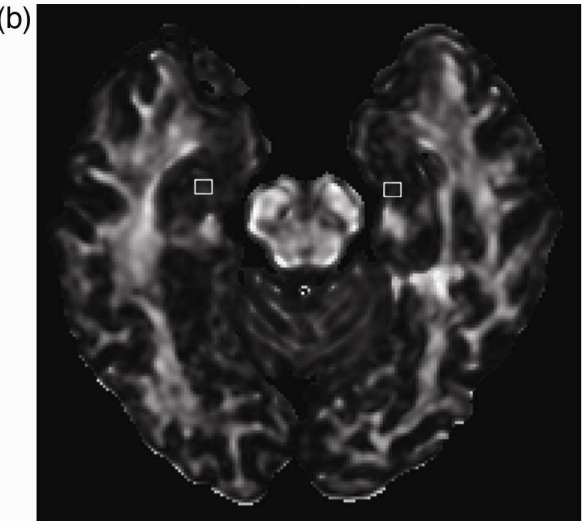

Fig. 3. Region of interest drawn in hippocampal area, from Iaria et al. (2008) figure 2. ROIs in the bilateral hippocampal regions are shown overlaid on diffusion images: (a) the colorcoded directionality maps (directions: red=left-right, green=anterior-posterior direction, blue=inferior-superior) and (b) monochromatic FA maps.

\section{(a)}

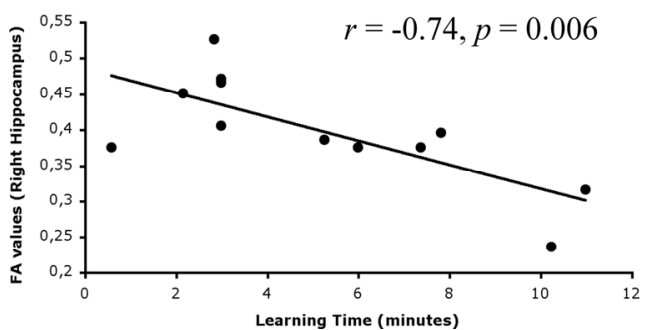

(b)

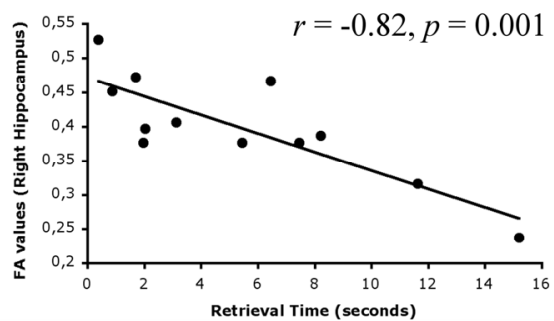

Fig. 4. FA value correlates with spatial navigation performance in healthy individuals, from Iaria et al. (2008) figure 3. A negative correlation was found between FA values of the right hippocampus and (a) the time spent by the participants to form a cognitive map of the environment (the learning task) and (b) the average time they spent to reach the target locations (the retrieval task). 


\subsection{Structural variation with age}

Diffusivity properties of cerebral white matter change across the lifespan of healthy individuals, likely reflecting developmental and aging-related changes in tract morphology (Bennett, Madden, Vaidya, Howard, \& Howard, 2010; Davis et al., 2009; Kochunov et al., 2010; Madden et al., 2009; Madden et al., 2007). Aging causes increases in MD and decreases in FA, presumably due to deterioration in the integrity of white matter tracts due to demyelination (Abe et al., 2002; Yoon, Shim, Lee, Shon, \& Yang, 2008). Recent studies have established an anterior-posterior gradient of age-related deterioration amongst healthy
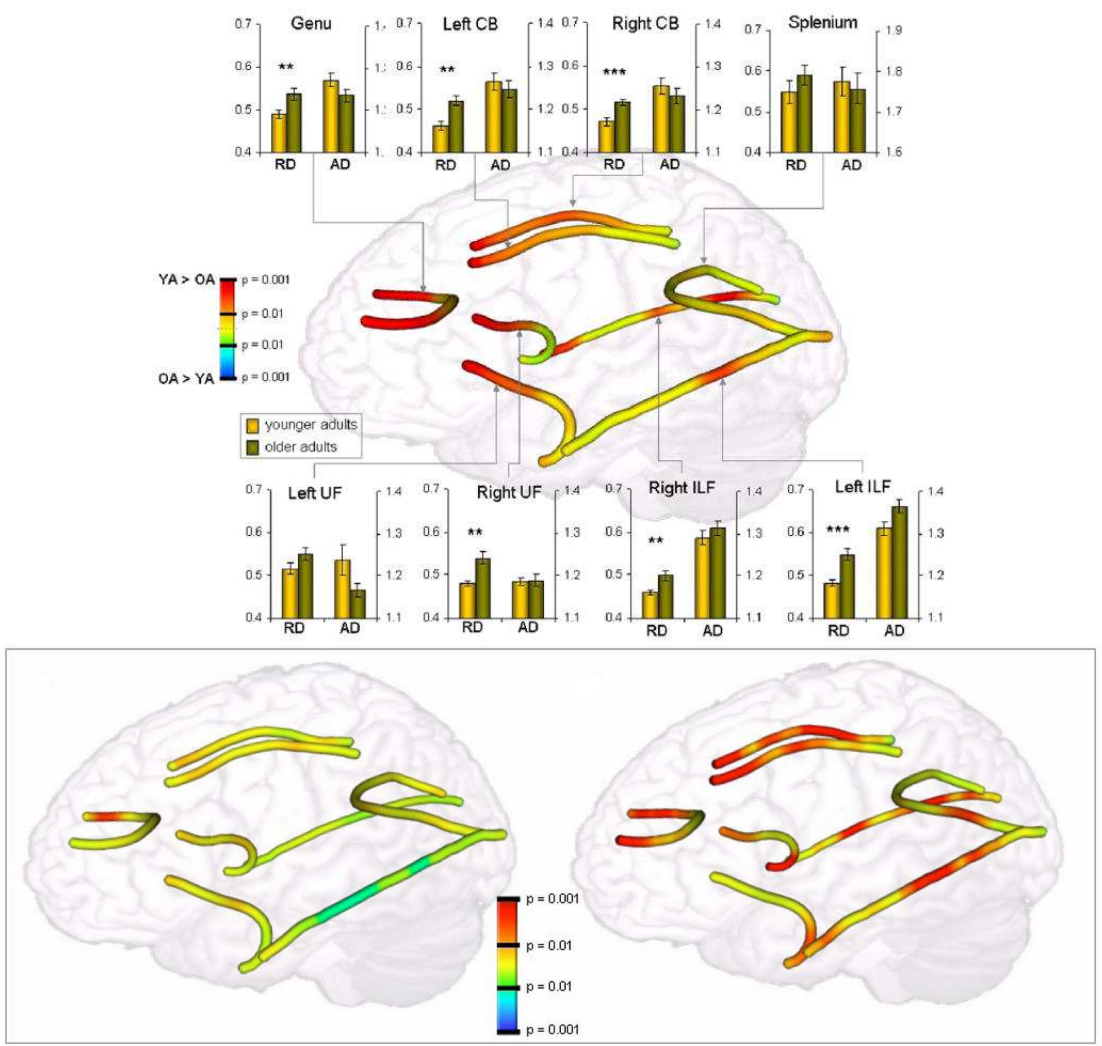

Fig. 5. Age-related differences in fractional anisotropy (FA), radial diffusivity (RD) and axial diffusivity (AD) for specific tracts, from Davis et al. (2009) figures 2 \& 3.

Top, FA differences are shown along a tract; FA data were smoothed with a $6 \mathrm{~mm}$ kernel and displayed upon mean tracts from a representative subject for the genu, uncinate fasciculi, cingulum bundle, inferior longitudinal fasciculi, and splenium. RD and AD differences are displayed in graphs corresponding to specific tracts. Metric dimensions: AD, RD: $10^{-3}$ $\mathrm{mm}^{2} / \mathrm{s} ;{ }^{*}=P<0.01 ;{ }^{* *}=\mathrm{P}<0.005 ; * * *=P<0.001$. YA $=$ younger adults; $\mathrm{OA}=$ older adults. Below, differences in $\mathrm{AD}$ (left) and RD (right) are represented upon the same tracts, demonstrating greater age-related effects for RD than AD. Colour scales for RD have been reversed such that OA > YA differences are reflected by warmer colors.

(Figures reproduced with permission from Elsevier) 
adults, such that indices of diffusivity show anterior regions of cortex are affected more, or perhaps sooner, than posterior regions (Bennett, et al., 2010; Davis, et al., 2009; Madden, et al., 2007; Yoon, et al., 2008). This anterior-posterior gradient of age impact is the reverse of the gradient observed in the childhood development of white and grey matter, where anterior regions have a more protracted development course than posterior regions (Eluvathingal, Hasan, Kramer, Fletcher, \& Ewing-Cobbs, 2007; Giedd et al., 1999; Paus et al., 1999; Sowell et al., 2004; Sowell, Trauner, Gamst, \& Jernigan, 2002). A current trend in DTI aging research is to examine radial diffusivity (RD) and axial diffusivity (AD), indices of diffusion in the primary and perpendicular directions of the diffusion tensor, which contribute to FA. AD describes the principal eigenvector of the diffusion tensor and is assumed to indicate the integrity of axons (Glenn et al., 2003) or changes in extraaxonal/extracellular space (Beaulieu \& Allen, 1994). RD describes an average of the eigenvectors that are perpendicular to the principal diffusion direction and is assumed to reflect changes associated with myelination or glial cell morphology (Song et al., 2003; Song et al., 2002; Song et al., 2005). Such techniques provide a unique insight into normal cortical changes across the lifespan that can also be correlated with associated behavioural changes.

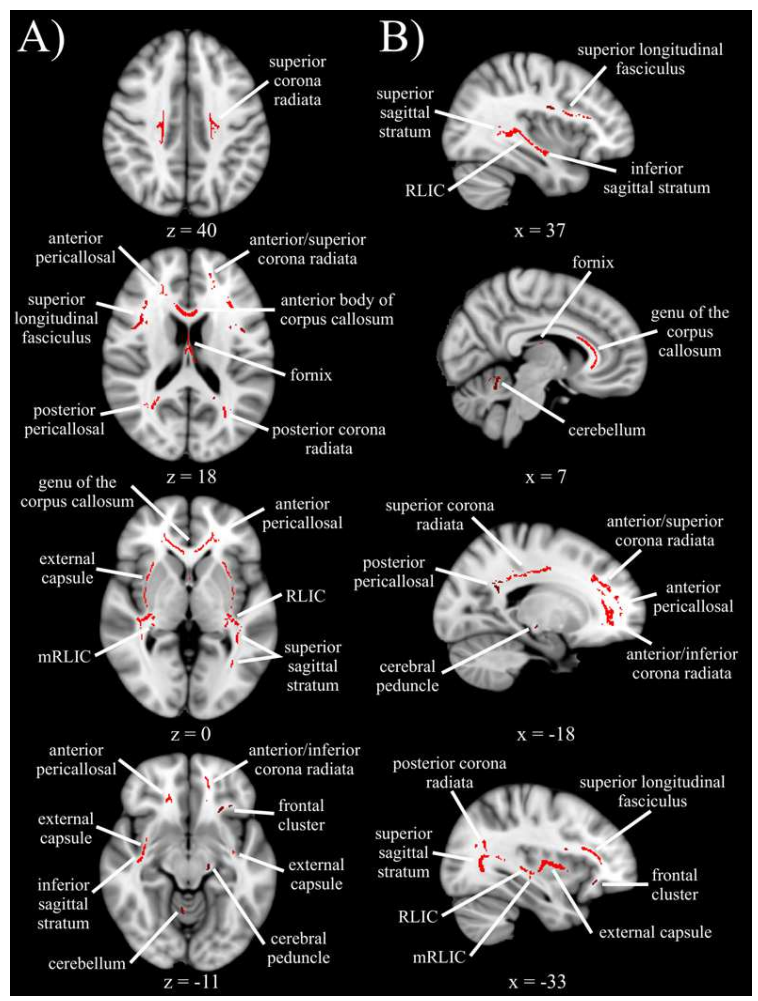

Fig. 6. FA differences across the brain related to aging, from Bennett et al. (2010) figure 1. Shown in red are white matter clusters where FA was significantly greater in younger compared to older adults, across four axial and four sagittal slices. Axial slices are presented in radiological orientation (right $=$ left). RLIC = retrolenticular part of the internal capsule. (Figure reproduced with permission from John Wiley and Sons) 
A recent study (Davis, et al., 2009) used streamline tractography (described later) to establish the location of several major white matter pathways in each individual's brain. Reconstructed tracts were then averaged to form a mean position for each pathway in each individual, and then combined into two groups: younger (mean age 20 years) and older (mean age 69 years) adults. In 10mm segments along each pathway, mean FA, AD and RD values were examined. Age-related differences were apparent in all three indices but especially the FA and AD. Consistent with the anterior-posterior model of FA changes with aging, the greatest age-related changes in FA occurred in anterior segments of longitudinal fibers that traversed the frontal lobe (see figure 5). In particular, examination of the cingulum bundle and uncinate fasciculus showed that age effects increase gradually from the posterior to anterior brain, likely due to myelodegeneration. In all cases age differences were greater for radial than axial diffusivity. In contrast, an age-related study (Bennett, et al., 2010) that examined FA changes throughout the brain rather than those within specified tracts, found that reduced FA values in older adults (as shown in figure 6) were caused by either an increase in RD only, increases in both RD and AD, or an increase in $\mathrm{RD}$ with a corresponding decrease in $\mathrm{AD}$, depending on region of the brain. Again, the largest changes were observed in frontal regions. The frontal cortex seems to be susceptible to early deterioration in dementia also. Changes in AD in tracts projecting to the frontal cortex have been associated with cases of amnestic mild cognitive impairment, a pre-cursor to Alzheimer's disease, with changes in diffusivity in all directions, and widespread FA decrease, apparent in cases of Alzheimer's disease (Agosta, et al., 2011).

\subsection{DTI in disease}

In addition to comparisons within the healthy population, DTI indices have been used to detect structural differences, and provide an index of disease state, in a range of neuropsychiatric and neurological diseases, for example multiple sclerosis (Sigal, Shmuel, Mark, Gil, \& Anat, 2010), Huntington's disease (Rosas et al., 2010), schizophrenia (Manoach et al., 2007), obsessive-compulsive disorder (Cannistraro et al., 2007), bipolar disorder (Adler et al., 2004; Beyer et al., 2005), Alzheimer's disease (Hanyu et al., 1997; Pievani et al., 2010), amnestic mild cognitive impairment (Agosta, et al., 2011), and in presymptomatic groups at risk of developing Alzheimer's disease (Gold, Powell, Andersen, \& Smith, 2010). Since anisotropy is modulated by the presence of myelin, diffusion indices have been used to demonstrate changes in white matter integrity in many diseases that involve myelin degeneration and, recently, as a way to measure neuroprotective therapies (R. J. Fox et al., 2011).

We used FA value to detect white matter deterioration in Niemann-Pick Type C (NPC) disease (Scheel, Abegg, Lanyon, Mattman, \& Barton, 2010). NPC is a fatal autosomal recessive neurodegenerative disorder resulting from mutations in the NPC1 or NPC2 genes (Vanier \& Millat, 2003) that leads to impaired intracellular lipid trafficking and consequent excess lysosomal storage of glycosphingolipids and cholesterol in multiple tissues. In the central nervous system, the storage material results in dysfunction and death of Purkinje and other select neuronal cells (Karten, Peake, \& Vance, 2009). The disorder affects the entire brain but some regions are particularly susceptible, with cerebellar degeneration leading to gait ataxia (a lack of coordination of muscle movements), dysarthria (motor speech disorder) and dysphagia (difficulty swallowing), and cortical degeneration leading to dementia and seizures. An early characteristic sign of the disease is vertical supranuclear gaze palsy, 
meaning that patients find it extremely difficult to make vertical eye movements, while their horizontal eye movements are nearer normal. These eye movement deficits are reflected in more severe cell loss in the rostral interstitial nucleus of the medial longitudinal fasciculus (riMLF), the nucleus linked to the generation of vertical eye movements, compared to the paramedian pontine reticular formation (PPRF), the nucleus linked to the generation of horizontal eye movements (Solomon, Winkelman, Zee, Gray, \& Buttner-Ennever, 2005). Standard clinical MR sequences are relatively insensitive to changes in the early stages of NPC. However, DTI, which measures the integrity of myelinated axons, is a plausible technique to identify early NPC changes, given the impaired lipid storage associated with the disease.

We examined FA values across the brain of a young early-stage NPC patient in comparison with a group of healthy control subjects. We found significantly lower FA in the patient compared to controls, particularly in white matter regions, as shown in figure 7. A voxelwise analysis, using a voxel-by-voxel statistical contrast (tract based spatial statistics tool of FSL: Smith et al., 2006) revealed some clusters of local FA differences. Of particular interest was a region of reduced FA in the dorsal midbrain because of the involvement of this region (riMLF) in the generation of vertical eye movements. Another prominent cluster was found in the superior cerebellar peduncle, a possible cause of the patient's cerebellar ataxia. After one year of treatment, areas of reduced FA persisted but there were significantly fewer voxels with lower FA and the cerebellar peduncle cluster was reduced in size, which might have been linked with the patient's improved tremor and dysarthria, and reports of fewer falls. Also, the midbrain cluster was not apparent after treatment, possibly correlating with the patient's slight improvement in making vertical eye movements.

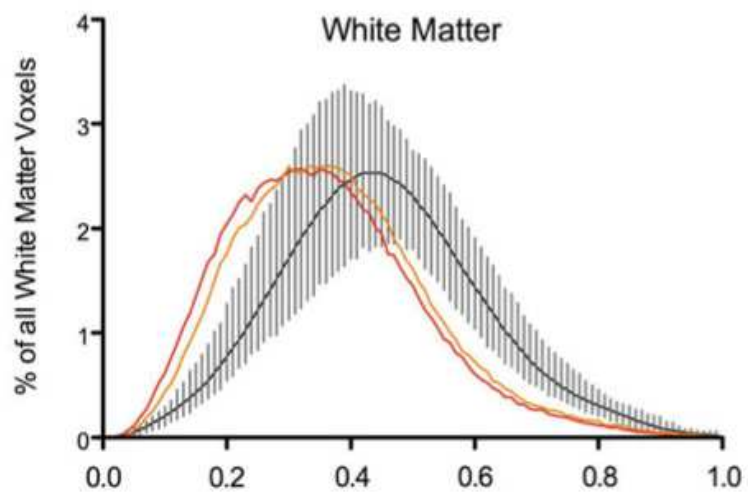

Fig. 7. Histogram of FA values for white matter regions, from Scheel et al. (2010) figure 2. Mean healthy FA value is shown as the black line with vertical errors bars representing the range of values. The NPC patient's FA value before treatment is shown (red line), and after 1 year of treatment with Miglustat (orange line). The patient's white matter has significantly lower FA value than that of healthy controls. The mean FA in the patient's white matter (0.359) was more than 6 standard deviations from the control's mean $(0.452 \pm 0.016)$. After treatment, the FA value improved to 0.377 , but was still 5 standard deviations from the control's mean. (Figure reproduced with permission from Elsevier) 
This example demonstrates the use of FA as an early marker of disease state (before abnormalities were evident in standard clinical MRI), revealing local changes that correlate with specific clinical signs. FA was also used as an objective structural index to track disease progression and assess the effects of treatment.

\section{Visualising tracts}

Assuming that the largest principal axis of the diffusion tensor aligns with the predominant fiber orientation in the MRI voxel, a vector field is created that reflects the orientation of fibers at all voxels throughout the brain. Tractography is the 3D reconstruction of tract trajectories from these vector fields. At the resolution of MRI voxels (typically 1-4mm for DTI), reconstructed tracts reflect the orientation of large white matter bundles present in the brain. The technique does not permit the tracking of individual axons or dendrites (whose typical size is $<10 \mu \mathrm{m}$ ). Hence, tractography models large-scale connectivity within the brain. It has provided insights into in vivo white matter morphology in health and disease/lesion. Methods of DTI tractography generally seek to propagate a line through the field using a deterministic algorithm based on tensor information at each stage of the propagation ${ }^{1}$. This is known as streamline tractography (Mori \& Barker, 1999; Mori, Crain, Chacko, \& van Zijl, 1999; Mori \& van Zijl, 2002). Simply, the vector field is converted into a continuous number field and a line is propagated through this field with a small step size, using the distanceaveraged vector information, as depicted in figure 8 . This algorithm is known as fiber assignment by continuous tracking (FACT) and was used for the first successful tract reconstruction, performed for a fixed rat brain (Mori, et al., 1999; Xue, van Zijl, Crain, Solaiyappan, \& Mori, 1999). From a starting seed voxel or ROI, tractography commences and lines are propagated while the FA value indicates high anisotropy (typically a threshold of 0.2 is chosen because voxels with lower FA value tend to be in grey matter or CSF). An example of fibers tracked from the striate cortex (area V1) is shown in figure 9.

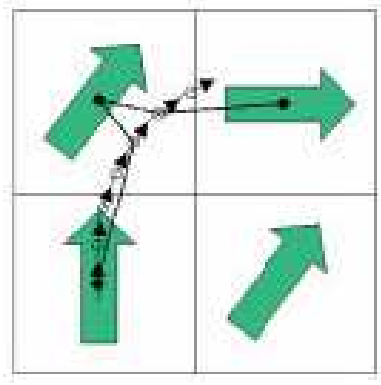

Fig. 8. FACT streamline technique for tractography, from Mori and van Zijl (2002). (Figure reproduced with permission from John Wiley and Sons)

\footnotetext{
1 An alternative approach to propagating a line thorough the tensor vector field is to use energy maximisation techniques (Parker, 2000). These techniques use principles of global energy minimisation to find the energetically most favourable path by which two points are connected. So far this approach has been less extensively adopted than streamline tractography and probabilistic methods.
} 


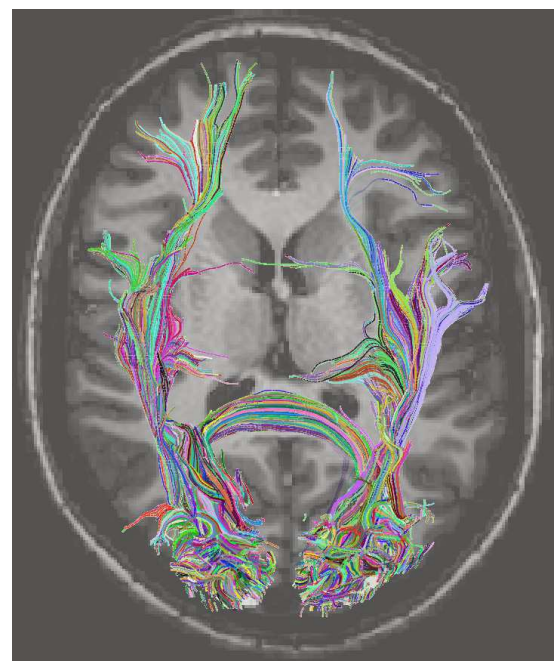

Fig. 9. Streamline tractography using primary visual cortex (area V1) as an ROI. An oval seed ROI was drawn over the occipital pole and streamline tractography performed in DTI Studio (Hiang \& Mori). Reconstructed tracts are shown in 3 dimensions overlaid on a 2 dimensional axial brain slice. Tracts forming part of the optic radiation and corpus callosum are some of the pathways shown. Note each tracked 'fiber' represents a line that has been tracked through the tensor vector field so each line represents multiple real axons and dendrites.

Once the tractography encounters a region of low anisotropy it ceases. An issue with this approach is that a DTI voxel is a few millimetres in size and more than one bundle of oriented tracts might be present, i.e. tracts of different orientations may cross within the voxel. If there are two predominant fiber directions within the voxel, the tensor may appear 'pancake-shaped' (see Mori \& van Zijl, 2002, for review) and, with higher numbers of directions the overall diffusion may appear isotropic. If the tractography is unable to determine the onward fiber direction, due to tensor isotropy, it stops at that voxel. Hence, tractography is difficult in regions of complex fiber architecture. Nonetheless, streamline tractography results are robust for major pathways and have been shown to have good correspondence with known neuroanatomy (Kang, Zhang, Carlson, \& Gembris, 2005; Mori \& van Zijl, 2002). To illustrate the potential and limitations of tractography, we will now discuss two studies where this form of deterministic streamline tractography was used to investigate pathways for visual perception in healthy humans.

\subsection{Pathways for visual motion perception}

Patients who sustain a loss of the primary visual cortex (striate area V1) are rendered blind in the respective part of their visual field. Most of these cortically blind patients have no residual visual perception. However, some "blindsight" patients (Weiskrantz, 1998) retain residual visual abilities such as the ability to make perceptual discriminations or perform associated visuomotor control at better than chance levels, while denying conscious awareness of stimuli in their blind visual fields (Blythe, Kennard, \& Ruddock, 1987; Corbetta, Marzi, Tassinari, \& Aglioti, 1990; Perenin \& Jeannerod, 1975; Perenin, Ruel, \& 
Hecaen, 1980). Several reports suggest that V1 is necessary for conscious visual perception (Celesia, Bushnell, Toleikis, \& Brigell, 1991; Cowey \& Stoerig, 1997; Merigan, Nealey, \& Maunsell, 1993). However, some degraded conscious "blindsight" vision may remain, particularly if structures like the superior colliculus, LGN and extrastriate cortex are not directly affected. Conscious perception of visual motion seems to be particularly likely to be spared (Barbur, Watson, Frackowiak, \& Zeki, 1993; Blythe, et al., 1987; Giaschi et al., 2003; Mestre, Brouchon, Ceccaldi, \& Poncet, 1992; Morland et al., 1999; Perenin, 1991; Ptito, Lepore, Ptito, \& Lassonde, 1991; Riddoch, 1917; Schoenfeld, Heinze, \& Woldroff, 2002), and motion perception has even been reported in a case where the striate cortex was completely congenitally absent (Giaschi, et al., 2003). Many cortical regions exhibit motion sensitivity, but key areas are the middle temporal area (MT), medial superior temporal cortex (MST) and the fundus of the superior temporal cortex (FST), known collectively in humans as the V5/MT complex, or V5/MT+ (Zeki et al., 1991). The main route by which V5/MT+ receives visual inputs from the retina is via the lateral geniculate nucleus (LGN) of the thalamus, which projects to $\mathrm{V} 1$ which, in turn, projects to V5/MT+. If this were the sole pathway by which visual motion is perceived, damage to any part of this path should render the person blind to visual motion. Human and animal studies suggest that there may be direct projections, perhaps from LGN, pulvinar or superior colliculus, to V5/MT+ that bypass V1 (Cowey \& Stoerig, 1991; Rodman, Gross, \& Albright, 1989; Stoerig \& Cowey, 1997). Such pathways might be responsible for the residual visual abilities observed in some cortically blind patients.

We used a combined functional magnetic resonance imaging (fMRI) and streamline tractography approach to investigate the presence of sub-cortical connections to V5/MT+ in 10 healthy human subjects (Lanyon, Giaschi et al., 2009). DTI data were collected using 32 gradient directions. We first localised V5/MT+ in each subject using fMRI (because the exact anatomical location of this area is subject to individual variation (Dumoulin et al., 2000; Watson et al., 1993) and then used this region as the seed for tractography. We performed all analyses in each subject's native space, without conversion to a standard brain template, in order to retain individual differences in tract morphology. Tracts extending to area V1 were eliminated from the analysis. We found individual variation in the results of tractography: direct connections between V5/MT+ and sub-cortical locations were found in 4 of our 10 subjects. All 4 of these subjects had fibers connecting V5/MT+ with the pulvinar, 2 had fibers connecting with the superior colliculus and 1 subject had fibers connecting with the region of the LGN. An example of fibers tracked to the pulvinar in one subject is given in figure 10. Further examples are given in Lanyon, Giaschi et al. (2009). Consistent with known feedback connections from V5/MT+ to the pons in monkeys (Boussaoud, Desimone, \& Ungerleider, 1992; Maunsell \& van Essen, 1983), fibers were found extending inferiorly in the brain stem through the pons in 3 of the 4 subjects, and a small number proceeded into the medulla.

Since the outputs of tractography are computationally-derived it is important to validate findings with other forms of evidence. Our tractography findings are supported by studies in monkeys: feedforward thalamic and collicular projections to V5/MT+ have been described (Boussaoud, et al., 1992; Cowey \& Stoerig, 1991, 1997; Cusick, Scripter, Darensbourg, \& Weber, 1993; Lin \& Kaas, 1980; Rockland, Andresen, Cowie, \& Robinson, 1999; Sincich, Park, Wohlgemuth, \& Horton, 2004; Stoerig \& Cowey, 1997); MST and FST have been shown to be reciprocally connected to the pulvinar, and to project to the pons (Boussaoud, et al., 1992); and feedback connections have been found from area MT to the 
pulvinar, LGN, the superior colliculus and the pons (Maunsell \& van Essen, 1983; Wall, Symonds, \& Kaas, 1982). DTI tractography can only confirm the presence of a hypothesised fiber tract, it does not provide information about the direction of axonal projections. Hence, the pathways we have identified could represent feedforward connections from thalamus to $\mathrm{V} 5 / \mathrm{MT}+$, or feedback from V5/MT+, or both. The evidence from monkeys supports both forms of connection.

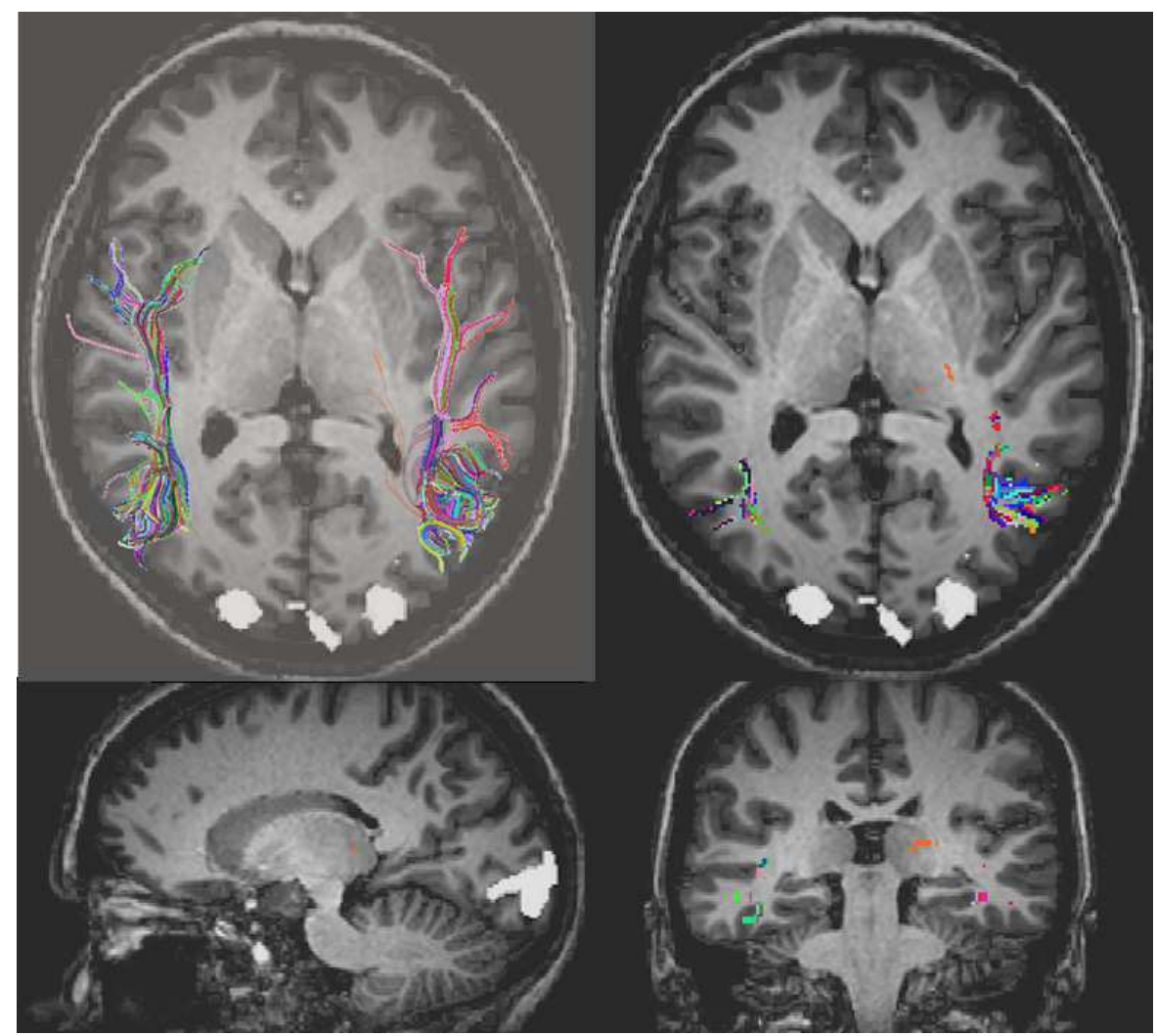

Fig. 10. Streamline tractography using functionally-defined visual cortical area V5/MT+ as an ROI, with fibers extending to area V1 eliminated.

Fibers extend from V5/MT+ to the pulvinar nucleus of the thalamus in the left hemisphere (orange line). The panels show output from DTI Studio (Hiang \& Mori). Top left:

reconstructed tracts are shown in 3 dimensions overlaid on a 2 dimensional axial brain slice. In the other panels only the fibers present in that slice are shown (top right: axial, bottom left: sagittal, bottom right: coronal views). Images are displayed in radiological convention $($ right $=$ left). White regions denote the location of functional activation in V1 and V5/MT+.

An example of a subject in which tractography did not connect V5/MT+ with subcortex is shown in figure 11. This inter-subject variability could be due to the technical limitations of DTI tractography in terms of resolving crossing fibers within a voxel, particularly since we were tracking through regions of complex fiber structure. However, the diversity in our 
results could also reflect true inter-subject anatomical variability of pathways, as suggested by others (Leh, Johansen-Berg, \& Ptito, 2006). Finding a direct pathway between subcortical structures and V5/MT+ in less than half our healthy subjects is consistent with the fact that only a minority of patients with cortical visual loss retain residual visual abilities (Barton \& Sharpe, 1997; Kasten, Wuest, \& Sabel, 1998; Scharli, Harman, \& Hogben, 1999).

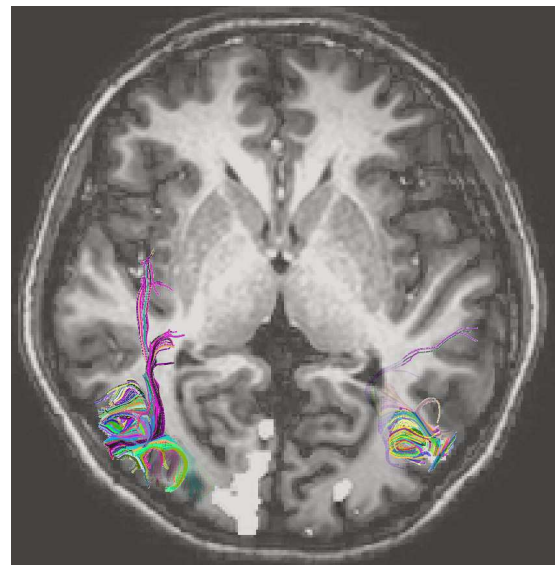

Fig. 11. Tractography in a subject for whom fibers did not connect V5/MT+ with subcortex.

\subsection{The network for face perception}

Functional MRI studies have identified multiple cortical regions that are involved in face perception, a key visuosocial skill (Gobbini \& Haxby, 2007; Haxby, et al., 2000; Ishai, et al., 2005; Rossion, et al., 2003). Current models divide these into a core system, which is predominantly involved in the processing of facial stimuli, and an extended system which, though not solely involved in face processing, contributes to it (Gobbini \& Haxby, 2007; Haxby, et al., 2000). The core system consists of the occipital face area (OFA), located on the inferior occipital gyrus; the fusiform face area (FFA), located on the lateral fusiform gyrus; and a face-selective region in the posterior superior temporal sulcus (pSTS) (Haxby, et al., 2000; Ishai, et al., 2005; Kanwisher, et al., 1997). These regions consistently show increased activity in fMRI studies that contrast activity to faces over other objects, with the right hemisphere being dominant for face processing. Damage to component parts of face processing cortical network can result in face processing deficits, a condition known as prosopagnosia (Barton, 2003; Rossion, et al., 2003). Patients with prosopagnosia are typically unable to recognize faces, a major social impediment. Prosopagnosia is a selective visual agnosia and the ability to recognize other objects is usually unimpaired. Prosopagnosia can take a developmental form, where onset occurs prior to the development of normal face recognition abilities (either due to genetic causes or acquired in early childhood), or be acquired in later life through cortical damage due to illness or injury. Various cortical lesion locations have been associated with the condition (see C. J. Fox et al., 2008, for a review). While loss of one or more of the functional components of the core face network is an obvious cause in some patients, for example a lesion in the area of the OFA in patient PS (Rossion, et al., 2003), other patients have robust fMRI activation in the three cortical areas of the core network. It has been suggested that some cases of prosopagnosia could be due to damage to the connectivity of the face processing network (C. J. Fox, et al., 2008). 
In order to better understand interconnectivity of the cortical face-processing network we performed DTI tractography in 5 healthy subjects and examined fibers tracked from OFA, FFA and pSTS, which were defined by fMRI. For the fMRI face-selective region localiser we contrasted activity invoked by presentation of static pictures of neutral and emotionally expressive faces with that evoked by presentation of static pictures of non-living objects (see C. J. Fox, Iaria, \& Barton, 2009, for fMRI methods). A threshold of $p<0.05$, Bonferonni corrected, was used to determine the extent of activation and this became the seed region for DTI tractography. DTI data were collected using 32 gradient directions. Structural, functional and DTI images were co-registered and streamline tractography was performed in Brain Voyager QX (www.brainvoyager.com, Brain Innovation) for each subject. All fMRI and DTI analysis were performed in each subject's own native brain space, without conversion to a standard template. Figure 12 shows fibers tracked from the regions of the core network in an example subject. Fibers from right OFA and FFA interconnected these regions and fibers from these posterior regions travelled anteriorally along the inferior longitudinal fasciculus toward the inferior temporal cortex (a region which forms part of the extended network, possibly contributing facial memories). We found a lack of fibers connecting right OFA, FFA and the right anterior temporal lobe in a prosopagnosic patient who had a right amygdalohippocampectomy for epilepsy that resulted in lesion of the anterior part of the inferior longitudinal fasciculus (Lanyon, Scheel, Fox, Iaria, \& Barton, 2009). Contrary to current models of the core cortical network for face perception (Haxby, et al., 2000), our tractography did not reveal a connection between pSTS and either FFA or OFA. However, we cannot rule out the existence of a connection between pSTS and these posterior regions because tractographic technical difficulty may have prevented fibers traversing this region, since it contains the intersection of several major fiber pathways.

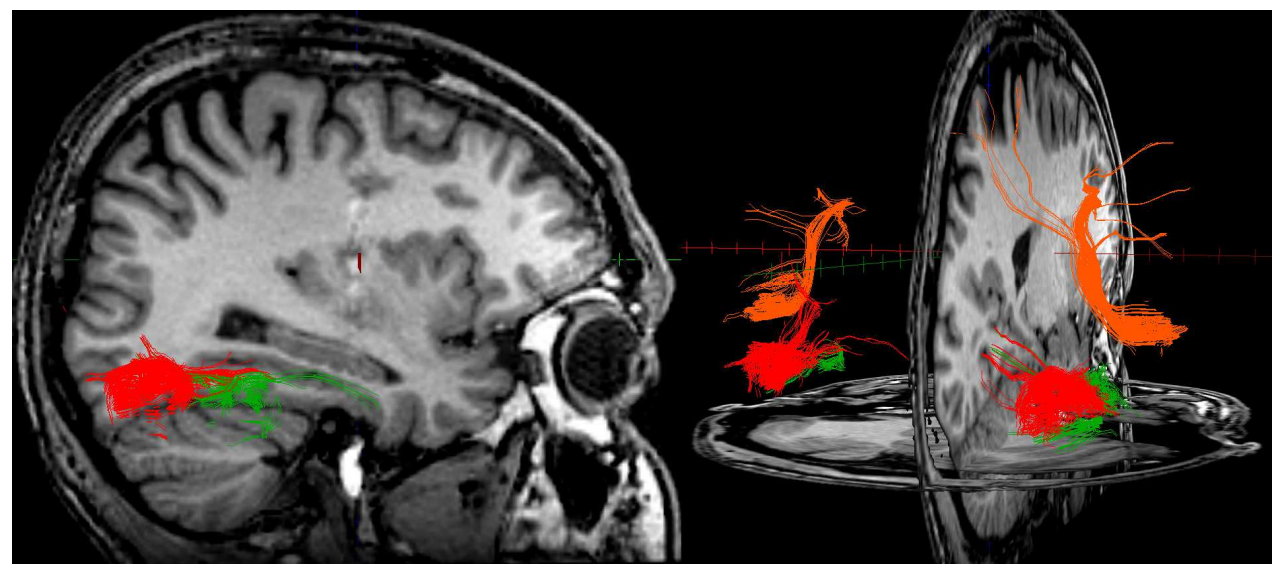

Fig. 12. Results of tractography showing fibers extending from core face-selective areas. The core network was defined by fMRI and then tractography performed using these areas as seed regions. OFA (red) FFA (green) and pSTS (orange) fibers in a healthy subject are shown. In the right hemisphere, fibers from OFA and FFA interconnect these two regions and extend along the inferior longitudinal fasciculus towards the inferior temporal cortex, as shown on the left. The three dimensional view on the right shows that the pSTS is not connected with OFA or FFA in our tractographic analysis. OFA = occipital face area; FFA = fusiform face area; pSTS = face-selective region in the posterior superior temporal sulcus. 


\subsection{Limitations and alternatives to the tensor model}

The tensor model of diffusion provided the first and currently most popular technique to map fiber orientations in the brain. It has proved to be a powerful model that has stimulated much research in the field of DW-MRI and adoption of these techniques. This has led to many insights into human neuroanatomy with visualisation of major fiber pathways in the brain. However, in fitting a Gaussian distribution of diffusion to each voxel, the tensor model assumes there is a single predominant orientation of fibers travelling within the voxel, and this results in the major limitation of DTI. In voxels containing complex fiber architecture, the tensor model cannot describe more than one dominant fiber orientation. Current research is attempting to provide models and tractography approaches that better account for the distribution of diffusion in voxels that contain crossing fibers. Many solutions rely on measuring diffusion over a higher number of angular directions. These techniques are referred to as high angular resolution diffusion imaging (HARDI). These highresolution methods aim to provide richer information about the direction of fibers in voxels that contain numerous fiber orientations.

\subsubsection{Diffusion Spectrum Imaging (DSI)}

DSI Diffusion spectrum imaging (DSI)(Wedeen, Hagmann, Tseng, Reese, \& Weisskoff, 2005; Wedeen et al., 2008) is a high angular technique that reconstructs a full account of fiber orientations by transforming data directly from the DW-MRI measurements. The result is an orientation distribution function (ODF). An ODF may be considered a deformed sphere whose radius in a given direction is proportional to the sum of values of the diffusion probability density function in that direction. The ODF has peaks in the directions in which the probability density function has most mass, which DSI assumes are the fiber directions. DSI is a superior technique to DTI in that DSI is able to resolve multiple fiber orientations. However, DSI is very time intensive during acquisition, because several hundreds of image volumes are required (compared to a minimum of 6 in DTI), and this has hindered its widespread use because it is often not well tolerated by subjects.

\subsubsection{Multi-compartmental models}

Models of diffusion that account for the multi-compartmental nature of matter within the voxel attempt to overcome the limitation of DTI imposed by the fitting of the single orientation Gaussian. Such models assume that each voxel comprises a discrete number of compartments reflecting particular fiber directions and extra-axonal components (Assaf, Freidlin, Rohde, \& Basser, 2004; Behrens et al., 2003). Hence, these models are an extension of DTI in which the tensor model is simply generalised to account for more than one fiber orientation, for example the single Gaussian model is replaced with a mixture of Gaussian densities. A problem with this approach is in deciding how many fiber orientations should be fitted, since selecting two or more can result in a loss of accuracy in voxels that contain a single predominant fiber orientation. A prominent model by Behrens et al. (2003) replaces the Gaussian model with a tensor that has only one nonzero eigenvalue and a Gaussian distribution in all other directions. Hence, a single fiber direction combined with isotropic diffusion in all other directions is modelled. Behrens et al. also take a probabilistic approach in which the likelihood of various fiber orientations is considered. This local probability distribution is then used to confer global probability estimations when determining whether two regions connect. This probabilistic tractography approach uses a sampling technique to 
draw samples based on the probability estimate of fibre direction at each voxel. Samples are generated from each voxel within the seed region and a connectivity distribution results in which the probability of a pathway passing through a voxel is reflected in the number of samples passing through it. Connections are visualised by applying a threshold to view only the most likely pathways from all calculated possible connection paths. Hence, local and global probability information is used to determine the likelihood of connections. Whereas streamline tractography traces fibers through regions of high anisotropy where uncertainty is low, these methods chose to model the uncertainty of fiber direction at each local voxel. Hence, it is claimed that this approach offers an advantage over streamline methods when tracking through regions of crossing fibers and orientation uncertainty. Typically tracts are converted to a standard anatomical template for group analysis and this carries some disadvantage in terms of a loss of individual information, given the high level of individual variation in morphology of both white and grey matter ${ }^{2}$.

\subsubsection{Estimators of the Orientation Distribution Function (ODF)}

A new generation of multiple-orientation fiber reconstruction algorithms is emerging. These attempt to combine the benefits of DTI (speed of acquisition) with the sophistication of the high angular DSI approach in estimating multiple fiber orientations. A distribution of fiber orientations within a voxel is assumed, rather than the discrete number assumed in the multi-compartmental approaches. Hence, these methods do not suffer from the problem of model selection since it is not required to know the number of fiber orientations in advance. Data is acquired using the spherical sampling scheme typical of DTI and the ODF is approximated by estimating the angular structure of the proton displacement probability density function directly from the MRI measurements. Examples of these approaches are q-ball imaging (Tuch, Reese, Wiegell, \& Wedeen, 2003), spherical deconvolution (Tournier, Calamante, Gadian, \& Connelly, 2004) and PASMRI (Jansons \& Alexander, 2003). These algorithms produce results in regions of fiber crossings that are in good agreement with DSI: see Alexander (2005) for a review of current approaches. However, these methods are currently computationally and acquisition-time heavy, and many still require implementation in software with a simple user interface to facilitate widespread adoption.

\subsection{Validation of results}

The outputs of tractography are very compelling and aesthetically interesting but results should be interpreted with some caution since tracked fibers are computational constructs and not imaged directly from the brain unlike chemically traced fibers, for example. Hence, it is normal practise for the results of DTI tractography to be compared with known findings from "gold standard" techniques such as chemical tract tracing in animal models and histological staining of brain slices. There will not necessarily be an exact correspondence of results, however, since DTI provides a level of macroscopic information that is difficult to obtain using chemical tracers in individual axons. DTI tractography then provides information that forms part of the converging evidence about brain connectivity. For

\footnotetext{
2 The issue of group analysis applies in general to all methods of tractography if fiber tracking is based upon ROIs defined by a group-level fMRI analysis since the location of these functionally-defined regions will extend into different regions of white matter in each individual and, hence, result in tracts being selected that would not be derived from an individual analysis.
} 
example, our tractographic identification of a pathway for visual motion perception is consistent not only with other DTI studies using alternative tractography techniques (Leh, Chakravarty, \& Ptito, 2008) but with the thalamic projections described in many histological and tracing studies, discussed above. Overall, tractography has proved to be remarkably accurate in reconstructing major fiber pathways in the human brain thought to exist based on evidence from post-mortem studies and animal models (Mori, et al., 1999).

\section{Conclusion}

Understanding how neural network connectivity confers human behaviour in health and disease is one of the most important challenges facing neuroscientists in the 21st century. DTI affords the ability to investigate the structure of large white matter pathways in vivo, offering profound insights into living human neural anatomy. In addition to its use in neuroscientific research, DTI as a method for detecting and monitoring certain disease states seems certain to become established in normal clinical practice. In this chapter we described a case of early Niemann-Pick Type C disease in which DTI revealed significant white matter structural changes which were not evident on standard clinical MRI structural imaging (Scheel, et al., 2010).

DTI offers an important contribution to the emerging array of scientific methods that aim to reveal insights into large-scale connectivity in the brain. Methods of fMRI functional connectivity (see Rogers, Morgan, Newton, \& Gore, 2007, for a review) confer information about connectivity between functionally active regions of the brain by examining temporal correlations in the fMRI signals in the active regions. Methods of effective connectivity additionally provide information about causal relationships, i.e. the direction of influence of one area upon another. Whereas DTI reveals structural connectivity in the brain, functional and effective connectivity methods show how these networks change under different stimulus or task conditions. Hence, DTI and functional/effective connectivity together can provide converging evidence about neural networks, from both a structural and a conditional (task- or stimulus-related) perspective. Such insights into structural and functional neural connectivity have not previously been possible in vivo. In addition, computational neuroscience models based on these empirical findings will provide a theoretical grounding for understanding neural network connectivity, as well as provoking further empirical investigation to answer questions deriving from these models.

Foremost amongst the key challenges in the development of DW-MRI methods is the development of algorithms capable of elucidating fiber directions in regions of complex structural architecture whilst being computationally tractable and remaining feasible in terms of acquisition times. The immediate future of DW-MRI research centres on improving methods for high angular resolution diffusion imaging so that acquisition and computation times are feasible. Techniques that exploit the full orientation distribution function of diffusion within each voxel will ultimately lead to better definition of fiber orientation in complex matter and, hence, provide more reliable descriptions of neural connectivity. To allow widespread adoption of these evolving technologies, it is necessary for the algorithms to be implemented in software platforms that can be readily used by medical and other researchers. As these new methods become more accessible in terms of acquisition times, computational tractability and general usability, DW-MRI will become an increasingly important method and even more pervasive in neuroscience research and in medical diagnosis and monitoring. DTI is just the beginning of this important field. 
"The human brain, then, is the most complicated organization of matter that we know"

Isaac Asimov

\section{Acknowledgments}

This work was supported by a Post-doctoral Fellowship from the Michael Smith Foundation for Health Research. Many thanks to my co-authors of various studies described in this chapter: Jason Barton MD PhD (to whom I extend additional thanks for providing the environment to study DTI), Debbie Giaschi PhD, Kevin Fitzpatrick BSc, Simon Au Young MSc, Giuseppe Iaria PhD, Michael Scheel MD, Mathias Abegg MD PhD, Christopher Fox $\mathrm{PhD}$, Bruce Bjornson MD, Lu Diao, Andre Mattman MD.

\section{References}

Abe, O., Aoki, S., Hayashi, N., Yamada, H., Kunimatsu, A., Mori, H., et al. (2002). Normal aging in the central nervous system: quantitative MR diffusion-tensor analysis. Neurobiology of Aging, 23(3), 433-441.

Adler, C. M., Holland, S. K., Schmithorst, V., Wilke, M., Weiss, K. L., Pan, H., et al. (2004). Abnormal frontal white matter tracts in bipolar disorder: a diffusion tensor imaging study. Bipolar Disorders, 6(3), 197-203.

Agosta, F., Pievani, M., Sala, S., Geroldi, C., Galluzzi, S., Frisoni, G. B., et al. (2011). White matter damage in Alzheimer disease and its relationship to gray matter atrophy. Radiology, 258(3), 853-863.

Alexander, D. C. (2005). Multiple-fiber reconstruction algorithms for diffusion MRI. Annals of the New York Academy of Sciences, 1064, 113-133.

Assaf, Y., Freidlin, R. Z., Rohde, G. K., \& Basser, P. J. (2004). New modeling and experimental framework to characterize hindered and restricted water diffusion in brain white matter. Magnetic Resonance in Medicine, 52(5), 965-978.

Barbur, J. L., Watson, J. D., Frackowiak, R. S., \& Zeki, S. (1993). Conscious visual perception without V1. Brain, 116 ( Pt 6), 1293-1302.

Barton, J. J. (2003). Disorders of face perception and recognition. Neurologic Clinics, 21(2), 521-548.

Barton, J. J., \& Sharpe, J. A. (1997). Smooth pursuit and saccades to moving targets in blind hemifields. A comparison of medial occipital, lateral occipital and optic radiation lesions. Brain, 120 ( Pt 4), 681-699.

Basser, P. J., \& Jones, D. K. (2002). Diffusion-tensor MRI: theory, experimental design and data analysis - a technical review. NMR in Biomedicine, 15(7-8), 456-467.

Basser, P. J., Mattiello, J., \& LeBihan, D. (1994). Estimation of the effective self-diffusion tensor from the NMR spin echo. Journal of Magnetic Resonance, Series B, 103(3), 247254.

Beaulieu, C. (2002). The basis of anisotropic water diffusion in the nervous system - a technical review. NMR in Biomedicine, 15(7-8), 435-455.

Beaulieu, C., \& Allen, P. S. (1994). Determinants of anisotropic water diffusion in nerves. Magnetic Resonance in Medicine, 31(4), 394-400. 
Behrens, T. E., Woolrich, M. W., Jenkinson, M., Johansen-Berg, H., Nunes, R. G., Clare, S., et al. (2003). Characterization and propagation of uncertainty in diffusion-weighted MR imaging. Magnetic resonance in medicine, 50(5), 1077-1088.

Bennett, I. J., Madden, D. J., Vaidya, C. J., Howard, D. V., \& Howard, J. H., Jr. (2010). Agerelated differences in multiple measures of white matter integrity: A diffusion tensor imaging study of healthy aging. Human Brain Mapping, 31(3), 378-390.

Berman, J. I., Mukherjee, P., Partridge, S. C., Miller, S. P., Ferriero, D. M., Barkovich, A. J., et al. (2005). Quantitative diffusion tensor MRI fiber tractography of sensorimotor white matter development in premature infants. NeuroImage, 27(4), 862-871.

Beyer, J. L., Taylor, W. D., MacFall, J. R., Kuchibhatla, M., Payne, M. E., Provenzale, J. M., et al. (2005). Cortical white matter microstructural abnormalities in bipolar disorder. Neuropsychopharmacology, 30(12), 2225-2229.

Blythe, I. M., Kennard, C., \& Ruddock, K. H. (1987). Residual vision in patients with retrogeniculate lesions of the visual pathways. Brain, 110, 887-905.

Boussaoud, D., Desimone, R., \& Ungerleider, L. G. (1992). Subcortical connections of visual areas MST and FST in macaques. Visual Neuroscience, 9(3-4), 291-302.

Cannistraro, P. A., Makris, N., Howard, J. D., Wedig, M. M., Hodge, S. M., Wilhelm, S., et al. (2007). A diffusion tensor imaging study of white matter in obsessive-compulsive disorder. Depression and Anxiety, 24(6), 440-446.

Celesia, G. G., Bushnell, D., Toleikis, S. C., \& Brigell, M. G. (1991). Cortical blindness and residual vision: is the "second" visual system in humans capable of more than rudimentary visual perception? Neurology, 41(6), 862-869.

Corbetta, M., Marzi, C. A., Tassinari, G., \& Aglioti, S. (1990). Effectiveness of different task paradigms in revealing blindsight. Brain, 113, 603-616.

Cowey, A., \& Stoerig, P. (1991). The neurobiology of blindsight. Trends in Neurosciences, 14(4), 140-145.

Cowey, A., \& Stoerig, P. (1997). Visual detection in monkeys with blindsight. Neuropsychologia, 35(7), 929-939.

Crank, J. (1975). The Mathematics of Diffusion. Oxford: Oxford University Press.

Cusick, C. G., Scripter, J. L., Darensbourg, J. G., \& Weber, J. T. (1993). Chemoarchitectonic subdivisions of the visual pulvinar in monkeys and their connectional relations with the middle temporal and rostral dorsolateral visual areas, MT and DLr. The Journal of Comparative Neurology, 336(1), 1-30.

Davis, S. W., Dennis, N. A., Buchler, N. G., White, L. E., Madden, D. J., \& Cabeza, R. (2009). Assessing the effects of age on long white matter tracts using diffusion tensor tractography. NeuroImage, 46(2), 530-541.

Dumoulin, S. O., Bittar, R. G., Kabani, N. J., Baker, C. L., Jr., Le Goualher, G., Bruce Pike, G., et al. (2000). A new anatomical landmark for reliable identification of human area V5/MT: a quantitative analysis of sulcal patterning. Cerebral Cortex, 10(5), 454-463.

Eluvathingal, T. J., Hasan, K. M., Kramer, L., Fletcher, J. M., \& Ewing-Cobbs, L. (2007). Quantitative diffusion tensor tractography of association and projection fibers in normally developing children and adolescents. Cerebral Cortex, 17(12), 2760-2768.

Fox, C. J., Iaria, G., \& Barton, J. J. (2008). Disconnection in prosopagnosia and face processing. Cortex, 44(8), 996-1009.

Fox, C. J., Iaria, G., \& Barton, J. J. (2009). Defining the face processing network: optimization of the functional localizer in fMRI. Human Brain Mapping, 30(5), 1637-1651. 
Fox, R. J., Cronin, T., Lin, J., Wang, X., Sakaie, K., Ontaneda, D., et al. (2011). Measuring myelin repair and axonal loss with diffusion tensor imaging. American Journal of Neuroradiology, 32(1), 85-91.

Giaschi, D., Jan, J. E., Bjornson, B., Young, S. A., Tata, M., Lyons, C. J., et al. (2003). Conscious visual abilities in a patient with early bilateral occipital damage. [Case Reports]. Developmental Medicine \& Child Neurology, 45(11), 772-781.

Giedd, J. N., Blumenthal, J., Jeffries, N. O., Castellanos, F. X., Liu, H., Zijdenbos, A., et al. (1999). Brain development during childhood and adolescence: a longitudinal MRI study. Nature Neuroscience, 2(10), 861-863.

Glenn, O. A., Henry, R. G., Berman, J. I., Chang, P. C., Miller, S. P., Vigneron, D. B., et al. (2003). DTI-based three-dimensional tractography detects differences in the pyramidal tracts of infants and children with congenital hemiparesis. Journal of Magnetic Resonance Imaging, 18(6), 641-648.

Gobbini, M. I., \& Haxby, J. V. (2007). Neural systems for recognition of familiar faces. Neuropsychologia, 45(1), 32-41.

Gold, B. T., Powell, D. K., Andersen, A. H., \& Smith, C. D. (2010). Alterations in multiple measures of white matter integrity in normal women at high risk for Alzheimer's disease. NeuroImage, 52(4), 1487-1494.

Hagmann, P., Jonasson, L., Maeder, P., Thiran, J. P., Wedeen, V. J., \& Meuli, R. (2006). Understanding diffusion MR imaging techniques: from scalar diffusion-weighted imaging to diffusion tensor imaging and beyond. RadioGraphics, 26, S205-223.

Hanyu, H., Shindo, H., Kakizaki, D., Abe, K., Iwamoto, T., \& Takasaki, M. (1997). Increased water diffusion in cerebral white matter in Alzheimer's disease. Gerontology, 43(6), 343-351.

Haxby, J. V., Hoffman, E. A., \& Gobbini, M. I. (2000). The distributed human neural system for face perception. Trends in Cognitive Sciences, 4(6), 223-233.

Hiang, H., \& Mori, S. Retrieved from http://lbam.med.jhmi.edu/DTIuser/DTIuser.asp.

Iaria, G., Lanyon, L. J., Fox, C. J., Giaschi, D., \& Barton, J. J. (2008). Navigational skills correlate with hippocampal fractional anisotropy in humans. Hippocampus, 18(4), 335-339.

Ishai, A., Schmidt, C. F., \& Boesiger, P. (2005). Face perception is mediated by a distributed cortical network. Brain Research Bulletin, 67(1-2), 87-93.

Jansons, K. M., \& Alexander, D. C. (2003). Persistent Angular Structure: new insights from diffusion MRI data. Information Processing in Medical Imaging 2003. Proceedings of the 18th International Conference (Vol. 18, pp. 672-683). Ambleside, United Kingdom.

Kang, N., Zhang, J., Carlson, E. S., \& Gembris, D. (2005). White matter fiber tractography via anisotropic diffusion simulation in the human brain. IEEE Transactions on Medical Imaging, 24(9), 1127-1137.

Kanwisher, N. (2010). Functional specificity in the human brain: a window into the functional architecture of the mind. Proceedings of the Nationall Academy of Science of the United States of America, 107(25), 11163-11170.

Kanwisher, N., McDermott, J., \& Chun, M. M. (1997). The fusiform face area: a module in human extrastriate cortex specialized for face perception. The Journal of Neuroscience, 17(11), 4302-4311. 
Karten, B., Peake, K. B., \& Vance, J. E. (2009). Mechanisms and consequences of impaired lipid trafficking in Niemann-Pick type C1-deficient mammalian cells. Biochimica et Biophysica Acta, 1791(7), 659-670.

Kasten, E., Wuest, S., \& Sabel, B. A. (1998). Residual vision in transition zones in patients with cerebral blindness. Journal of Clinical and Experimental Neuropsychology, 20(5), 581-598.

Klingberg, T., Hedehus, M., Temple, E., Salz, T., Gabrieli, J. D., Moseley, M. E., et al. (2000). Microstructure of temporo-parietal white matter as a basis for reading ability: evidence from diffusion tensor magnetic resonance imaging. Neuron, 25(2), 493-500.

Kochunov, P., Williamson, D. E., Lancaster, J., Fox, P., Cornell, J., Blangero, J., et al. (2010). Fractional anisotropy of water diffusion in cerebral white matter across the lifespan. Neurobiology of Aging, In Press.

Kwong, K. K., Belliveau, J. W., Chesler, D. A., Goldberg, I. E., Weisskoff, R. M., Poncelet, B. P., et al. (1992). Dynamic magnetic resonance imaging of human brain activity during primary sensory stimulation. Proceedings of the Nationall Academy of Science of the United States of America, 89(12), 5675-5679.

Lanyon, L. J., Giaschi, D., Young, S. A., Fitzpatrick, K., Diao, L., Bjornson, B. H., et al. (2009). Combined functional MRI and diffusion tensor imaging analysis of visual motion pathways. Journal of Neuro-Ophthalmology, 29(2), 96-103.

Lanyon, L. J., Scheel, M., Fox, C. J., Iaria, G., \& Barton, J. J. S. (2009). Disconnection of cortical face network in prosopagnosia revealed by diffusion tensor imaging Journal of Vision, 9(8), 482.

Lauterbur, P. C. (1973). Image formation by induced local interactions - examples employing nuclear magnetic-resonance. Nature, 242(5394), 190-191.

Le Bihan, D. (2003). Looking into the functional architecture of the brain with diffusion MRI. Nature Reviews Neuroscience, 4(6), 469-480.

Le Bihan, D., Breton, E., Lallemand, D., Grenier, P., Cabanis, E., \& Laval-Jeantet, M. (1986). MR imaging of intravoxel incoherent motions: application to diffusion and perfusion in neurologic disorders. Radiology, 161(2), 401-407.

Leh, S. E., Chakravarty, M. M., \& Ptito, A. (2008). The connectivity of the human pulvinar: a diffusion tensor imaging tractography study. International Journal of Biomedical Imaging, 2008, 789539.

Leh, S. E., Johansen-Berg, H., \& Ptito, A. (2006). Unconscious vision: new insights into the neuronal correlate of blindsight using diffusion tractography. Brain, 129(Pt 7), 18221832.

Lin, C. S., \& Kaas, J. H. (1980). Projections from the medial nucleus of the inferior pulvinar complex to the middle temporal area of the visual cortex. Neuroscience, 5(12), 22192228.

Madden, D. J., Spaniol, J., Costello, M. C., Bucur, B., White, L. E., Cabeza, R., et al. (2009). Cerebral white matter integrity mediates adult age differences in cognitive performance. Journal of Cognitive Neuroscience, 21(2), 289-302.

Madden, D. J., Spaniol, J., Whiting, W. L., Bucur, B., Provenzale, J. M., Cabeza, R., et al. (2007). Adult age differences in the functional neuroanatomy of visual attention: A combined fMRI and DTI study. Neurobiology of Aging, 28(3), 459-476.

Manoach, D. S., Ketwaroo, G. A., Polli, F. E., Thakkar, K. N., Barton, J. J., Goff, D. C., et al. (2007). Reduced microstructural integrity of the white matter underlying anterior 
cingulate cortex is associated with increased saccadic latency in schizophrenia. NeuroImage, 37(2), 599-610.

Maunsell, J. H., \& van Essen, D. C. (1983). The connections of the middle temporal visual area (MT) and their relationship to a cortical hierarchy in the macaque monkey. The Journal of Neuroscience, 3(12), 2563-2586.

Merboldt, K. D., Hanicke, W., \& Frahm, J. (1985). Self-diffusion NMR imaging using stimulated echoes. Journal of Magnetic Resonance, 64(3), 479-486.

Merigan, W. H., Nealey, T. A., \& Maunsell, J. H. (1993). Visual effects of lesions of cortical area V2 in macaques. The Journal of Neuroscience, 13(7), 3180-3191.

Mestre, D. R., Brouchon, M., Ceccaldi, M., \& Poncet, M. (1992). Perception of optical-flow in cortical blindness - a case report. Neuropsychologia, 30(9), 783-795.

Mori, S., \& Barker, P. B. (1999). Diffusion magnetic resonance imaging: its principle and applications. The Anatomical Record, 257(3), 102-109.

Mori, S., Crain, B. J., Chacko, V. P., \& van Zijl, P. C. (1999). Three-dimensional tracking of axonal projections in the brain by magnetic resonance imaging. Annals of Neurology, 45(2), 265-269.

Mori, S., \& van Zijl, P. C. (2002). Fiber tracking: principles and strategies - a technical review. NMR in Biomedicine, 15(7-8), 468-480.

Morland, A. B., Jones, S. R., Finlay, A. L., Deyzac, E., Le, S., \& Kemp, S. (1999). Visual perception of motion, luminance and colour in a human hemianope. Brain, 122 ( Pt 6), 1183-1198.

Moseley, M. E., Kucharczyk, J., Mintorovitch, J., Cohen, Y., Kurhanewicz, J., Derugin, N., et al. (1990). Diffusion-weighted MR imaging of acute stroke: correlation with T2weighted and magnetic susceptibility-enhanced MR imaging in cats. American Journal of Neuroradiology, 11(3), 423-429.

Ogawa, S., Tank, D. W., Menon, R., Ellermann, J. M., Kim, S. G., Merkle, H., et al. (1992). Intrinsic signal changes accompanying sensory stimulation: functional brain mapping with magnetic resonance imaging. Proceedings of the Nationall Academy of Science of the United States of America, 89(13), 5951-5955.

Ohnishi, T., Matsuda, H., Hirakata, M., \& Ugawa, Y. (2006). Navigation ability dependent neural activation in the human brain: an fMRI study. Neuroscience Research, 55(4), 361-369.

Parker, G. J. (2000). Tracing fiber tracts using fast marching. Paper presented at the International Society of Magnetic Resonance, Denver, CO.

Paus, T., Zijdenbos, A., Worsley, K., Collins, D. L., Blumenthal, J., Giedd, J. N., et al. (1999). Structural maturation of neural pathways in children and adolescents: in vivo study. Science, 283(5409), 1908-1911.

Perenin, M. T. (1991). Discrimination of motion direction in perimetrically blind fields. NeuroReport, 2(7), 397-400.

Perenin, M. T., \& Jeannerod, M. (1975). Residual vision in cortically blind hemifields. Neuropsychologia, 13(1), 1-7.

Perenin, M. T., Ruel, J., \& Hecaen, H. (1980). Residual visual capacities in a case of cortical blindness. Cortex, 16(4), 605-612.

Pierpaoli, C., Jezzard, P., Basser, P. J., Barnett, A., \& Di Chiro, G. (1996). Diffusion tensor MR imaging of the human brain. Radiology, 201(3), 637-648. 
Pievani, M., Agosta, F., Pagani, E., Canu, E., Sala, S., Absinta, M., et al. (2010). Assessment of white matter tract damage in mild cognitive impairment and Alzheimer's disease. Human Brain Mapping, 31(12), 1862-1875.

Ptito, A., Lepore, F., Ptito, M., \& Lassonde, M. (1991). Target detection and movement discrimination in the blind field of hemispherectomized patients. Brain, 114, 497512.

Riddoch, G. (1917). Dissociation of visual perceptions due to occipital injuries, with especial reference to appreciation of movement. Brain, 40, 15-57.

Rockland, K. S., Andresen, J., Cowie, R. J., \& Robinson, D. L. (1999). Single axon analysis of pulvinocortical connections to several visual areas in the macaque. The Journal of Comparative Neurology, 406(2), 221-250.

Rodman, H. R., Gross, C. G., \& Albright, T. D. (1989). Afferent basis of visual response properties in area MT of the macaque. I. Effects of striate cortex removal. The Journal of Neuroscience, 9(6), 2033-2050.

Rogers, B. P., Morgan, V. L., Newton, A. T., \& Gore, J. C. (2007). Assessing functional connectivity in the human brain by fMRI. Magnetic Resonance Imaging, 25(10), 13471357.

Rosas, H. D., Lee, S. Y., Bender, A. C., Zaleta, A. K., Vangel, M., Yu, P., et al. (2010). Altered white matter microstructure in the corpus callosum in Huntington's disease: Implications for cortical "disconnection". NeuroImage, 49(4), 2995-3004.

Rossion, B., Caldara, R., Seghier, M., Schuller, A. M., Lazeyras, F., \& Mayer, E. (2003). A network of occipito-temporal face-sensitive areas besides the right middle fusiform gyrus is necessary for normal face processing. Brain, 126(Pt 11), 2381-2395.

Scharli, H., Harman, A. M., \& Hogben, J. H. (1999). Blindsight in subjects with homonymous visual field defects. Journal of Cognitive Neuroscience, 11(1), 52-66.

Scheel, M., Abegg, M., Lanyon, L. J., Mattman, A., \& Barton, J. J. (2010). Eye movement and diffusion tensor imaging analysis of treatment effects in a Niemann-Pick Type C patient. Molecular Genetics and Metabolism, 99(3), 291-295.

Schoenfeld, M. A., Heinze, H. J., \& Woldroff, M. G. (2002). Unmasking motion-processing activity in human brain area V5/MT+ mediated by pathways that bypass primary visual cortex. NeuroImage, 17(2), 769-779.

Sigal, T., Shmuel, M., Mark, D., Gil, H., \& Anat, A. (2010). Diffusion tensor imaging of corpus callosum integrity in multiple sclerosis: Correlation with disease variables. Journal of Neuroimaging, in press. Epub ahead of print doi:10.1111/j.15526569.2010.00556.x.

Sincich, L. C., Park, K. F., Wohlgemuth, M. J., \& Horton, J. C. (2004). Bypassing V1: a direct geniculate input to area MT. Nature Neuroscience, 7(10), 1123-1128.

Smith, S. M., Jenkinson, M., Johansen-Berg, H., Rueckert, D., Nichols, T. E., Mackay, C. E., et al. (2006). Tract-based spatial statistics: Voxelwise analysis of multi-subject diffusion data. NeuroImage, 31(4), 1487-1505.

Solomon, D., Winkelman, A. C., Zee, D. S., Gray, L., \& Buttner-Ennever, J. (2005). NiemannPick type $C$ disease in two affected sisters: ocular motor recordings and brain-stem neuropathology. Annals of the New York Academy of Sciences, 1039, 436-445.

Song, S. K., Sun, S. W., Ju, W. K., Lin, S. J., Cross, A. H., \& Neufeld, A. H. (2003). Diffusion tensor imaging detects and differentiates axon and myelin degeneration in mouse optic nerve after retinal ischemia. NeuroImage, 20(3), 1714-1722. 
Song, S. K., Sun, S. W., Ramsbottom, M. J., Chang, C., Russell, J., \& Cross, A. H. (2002). Dysmyelination revealed through MRI as increased radial (but unchanged axial) diffusion of water. NeuroImage, 17(3), 1429-1436.

Song, S. K., Yoshino, J., Le, T. Q., Lin, S. J., Sun, S. W., Cross, A. H., et al. (2005). Demyelination increases radial diffusivity in corpus callosum of mouse brain. NeuroImage, 26(1), 132-140.

Sowell, E. R., Thompson, P. M., Leonard, C. M., Welcome, S. E., Kan, E., \& Toga, A. W. (2004). Longitudinal mapping of cortical thickness and brain growth in normal children. The Journal of Neuroscience, 24(38), 8223-8231.

Sowell, E. R., Trauner, D. A., Gamst, A., \& Jernigan, T. L. (2002). Development of cortical and subcortical brain structures in childhood and adolescence: a structural MRI study. Developmental Medicine \& Child Neurology, 44(1), 4-16.

Stoerig, P., \& Cowey, A. (1997). Blindsight in man and monkey. Brain, 120, 535-559.

Taylor, D. G., \& Bushell, M. C. (1985). The spatial mapping of translational diffusion coefficients by the NMR imaging technique. Physics in Medicine and Biology, 30(4), 345-349.

Tolman, E. C. (1948). Cognitive maps in rats and men. Psychological Review, 55, 189-208.

Tournier, J. D., Calamante, F., Gadian, D. G., \& Connelly, A. (2004). Direct estimation of the fiber orientation density function from diffusion-weighted MRI data using spherical deconvolution. NeuroImage, 23(3), 1176-1185.

Tuch, D. S., Reese, T. G., Wiegell, M. R., \& Wedeen, V. J. (2003). Diffusion MRI of complex neural architecture. Neuron, 40(5), 885-895.

Tuch, D. S., Salat, D. H., Wisco, J. J., Zaleta, A. K., Hevelone, N. D., \& Rosas, H. D. (2005). Choice reaction time performance correlates with diffusion anisotropy in white matter pathways supporting visuospatial attention. Proceedings of the National Academy of Sciences of the United States of America, 102(34), 12212-12217.

Vanier, M. T., \& Millat, G. (2003). Niemann-Pick disease type C. Clinical Genetics, 64(4), 269281.

Wall, J. T., Symonds, L. L., \& Kaas, J. H. (1982). Cortical and subcortical projections of the middle temporal area (MT) and adjacent cortex in galagos. The Journal of Comparative Neurology, 211(2), 193-214.

Watson, J. D., Myers, R., Frackowiak, R. S., Hajnal, J. V., Woods, R. P., Mazziotta, J. C., et al. (1993). Area V5 of the human brain: evidence from a combined study using positron emission tomography and magnetic resonance imaging. Cerebral Cortex, 3(2), 79-94.

Wedeen, V. J., Hagmann, P., Tseng, W. Y. I., Reese, T. G., \& Weisskoff, R. M. (2005). Mapping complex tissue architecture with diffusion spectrum magnetic resonance imaging. Magnetic Resonance in Medicine, 54(6), 1377-1386.

Wedeen, V. J., Wang, R. P., Schmahmann, J. D., Benner, T., Tseng, W. Y., Dai, G., et al. (2008). Diffusion spectrum magnetic resonance imaging (DSI) tractography of crossing fibers. NeuroImage, 41(4), 1267-1277.

Weiskrantz, L. (1998). Consciousness and commentaries Toward a Science of Consciousness II Second Tucson Discussions and Debates 1996 (pp. 371-377). University of Arisona, Tucson, AZ, USA: MIT Press, Cambridge, MA. 
Wolbers, T., Schoell, E. D., \& Buchel, C. (2006). The predictive value of white matter organization in posterior parietal cortex for spatial visualization ability. NeuroImage, 32(3), 1450-1455.

Xue, R., van Zijl, P. C., Crain, B. J., Solaiyappan, M., \& Mori, S. (1999). In vivo threedimensional reconstruction of rat brain axonal projections by diffusion tensor imaging. Magnetic Resonance in Medicine, 42(6), 1123-1127.

Yoon, B., Shim, Y. S., Lee, K. S., Shon, Y. M., \& Yang, D. W. (2008). Region-specific changes of cerebral white matter during normal aging: a diffusion-tensor analysis. Archives of Gerontology and Geriatrics, 47(1), 129-138.

Zeki, S., Watson, J. D., Lueck, C. J., Friston, K. J., Kennard, C., \& Frackowiak, R. S. (1991). A direct demonstration of functional specialization in human visual cortex. The Journal of Neuroscience, 11(3), 641-649. 


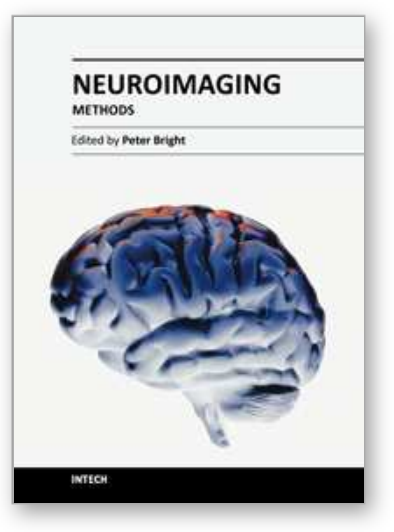

\author{
Neuroimaging - Methods \\ Edited by Prof. Peter Bright
}

ISBN 978-953-51-0097-3

Hard cover, 358 pages

Publisher InTech

Published online 17, February, 2012

Published in print edition February, 2012

Neuroimaging methodologies continue to develop at a remarkable rate, providing ever more sophisticated techniques for investigating brain structure and function. The scope of this book is not to provide a comprehensive overview of methods and applications but to provide a 'snapshot' of current approaches using well established and newly emerging techniques. Taken together, these chapters provide a broad sense of how the limits of what is achievable with neuroimaging methods are being stretched.

\title{
How to reference
}

In order to correctly reference this scholarly work, feel free to copy and paste the following:

Linda J. Lanyon (2012). Diffusion Tensor Imaging: Structural Connectivity Insights, Limitations and Future Directions, Neuroimaging - Methods, Prof. Peter Bright (Ed.), ISBN: 978-953-51-0097-3, InTech, Available from: http://www.intechopen.com/books/neuroimaging-methods/diffusion-tensor-imaging-structuralconnectivity-insights-limitations-and-future-directions

\section{INTECH}

open science | open minds

\section{InTech Europe}

University Campus STeP Ri

Slavka Krautzeka 83/A

51000 Rijeka, Croatia

Phone: +385 (51) 770447

Fax: +385 (51) 686166

www.intechopen.com

\section{InTech China}

Unit 405, Office Block, Hotel Equatorial Shanghai

No.65, Yan An Road (West), Shanghai, 200040, China

中国上海市延安西路65号上海国际贵都大饭店办公楼 405 单元

Phone: +86-21-62489820

Fax: $+86-21-62489821$ 
(C) 2012 The Author(s). Licensee IntechOpen. This is an open access article distributed under the terms of the Creative Commons Attribution 3.0 License, which permits unrestricted use, distribution, and reproduction in any medium, provided the original work is properly cited. 